\title{
THE MINIMUM MODULUS OF GAP POWER SERIES
}

\author{
by P. C. FENTON \\ (Received 12th October 1976)
}

\section{Introduction}

Let

$$
f(z)=\sum_{n=0}^{\infty} a_{n} z^{\lambda_{n}}
$$

be an entire function, where $\left(\lambda_{n}\right)$ is a strictly increasing sequence of non-negative integers. The maximum modulus, $M(r)$, the minimum modulus, $m(r)$, and the maximum term, $\mu(r)$, of $f$ are defined by

$$
M(r)=\max _{|z|=r}|f(z)|, \quad m(r)=\min _{|z|=r}|f(z)|, \quad \mu(r)=\max _{n \geq 0}\left|a_{n}\right| r^{\lambda_{n}} .
$$

The largest number $\lambda_{n}$ for which $\mu(r)=\left|a_{n}\right| r^{\lambda_{n}}$ is called the central index, $N(r)$, of $f$.

We recall briefly the notions of logarithmic measure and logarithmic density of linear sets. Given a set $E$ of non-negative numbers, the logarithmic measure of $E$ is defined by

$$
\log \text { meas } E=\int_{E n(1, \infty)} \frac{d t}{t} .
$$

The upper logarithmic density, $\overline{\log \text { dens }} E$, and the lower logarithmic density, $\log$ dens $E$, of $E$ are defined by

$$
\overline{\log \operatorname{dens}}=\varlimsup_{r \rightarrow \infty}(\log r)^{-1} \int_{E(1, r)} \frac{d t}{t} .
$$

Should the two limits of (1.2) prove to be equal we speak simply of the logarithmic density of $E$.

In (1), P. Erdös and A. J. Macintyre established conditions under which

$$
\varlimsup_{r \rightarrow \infty} \frac{m(r)}{M(r)}=\varlimsup_{r \rightarrow \infty} \frac{\mu(r)}{M(r)}=1
$$

for the entire function (1.1). Their results ((1) Theorems 1 and 4) may be gathered as follows:

(a) If $\sum_{k=0}^{\infty} \frac{1}{\lambda_{k+1}-\lambda_{k}}<\infty$; or

(b) if $\sum_{k=0}^{\infty} \frac{1}{\lambda_{k+1}-\lambda_{k}}=o\left(\log \lambda_{n}\right)$ and $f$ is of finite order; or 
(c) if $\sum_{k=0}^{n} \frac{1}{\lambda_{k+1}-\lambda_{k}}=O\left(\log \lambda_{n}\right)$ and $f$ is of zero order;

then (1.3) holds.

It is the purpose of this note to prove a sharpened version of this result both by establishing an estimate of the size of the set on which, as $r \rightarrow \infty$,

$$
\mu(r)=(1+o(1)) M(r), \quad m(r)=(1+o(1)) M(r)
$$

hold together and also by relaxing slightly the conditions (b) and (c).

\section{The result}

We will prove

(i) If $\sum_{k=0}^{\infty}\left(1 / \lambda_{k+1}-\lambda_{k}\right)<\infty$, then (1.4) hold together as $r$ tends to infinity outside a set of finite logarithmic measure.

(ii) If $\underline{\lim }_{n \rightarrow \infty}\left(\log \lambda_{n}\right)^{-1} \sum_{k=0}^{n}\left(1 / \lambda_{k+1}-\lambda_{k}\right)=0$ and $f$ is of finite order; or

(iii) if $\lim _{n \rightarrow \infty}\left(\log \lambda_{n}\right)^{-1} \sum_{k=0}^{n}\left(1 / \lambda_{k+1}-\lambda_{k}\right)<\infty$ and $f$ is of zero order; then (1.4) hold together as $r$ tends to infinity outside a set of zero lower logarithmic density.

Erdös and Macintyre show by counterexamples that if any one of the hypotheses concerning the indices $\lambda_{n}$ in (i), (ii) and (iii) is false then there is an entire function of the form (1.1) of the appropriate order (that is, of finite order in case (ii) and of zero order in case (iii)) for which both

$$
\varlimsup_{r \rightarrow \infty} \frac{m(r)}{M(r)} \leqslant 1 / 2, \quad \varlimsup_{r \rightarrow \infty} \frac{\mu(r)}{M(r)} \leqslant 1 / 2
$$

In this sense, then, the result is best possible.

\section{Preliminaries and a lemma}

Let

$$
\epsilon_{n}=\frac{1}{\lambda_{n+1}-\lambda_{n}}, \quad n=0,1,2, \ldots
$$

Given any non-negative integer $n$, let $n_{0}=n_{0}(n)$ be such that

$$
\lambda_{n_{0}} \leqslant 2 \lambda_{n}<\lambda_{n_{0}+1}
$$

and let us define a number $\delta_{n}$ by

$$
\delta_{n}=\max _{i \leqslant n \leqslant j \leqslant n_{0}}(j-i+1)^{-3 / 2} \sum_{\nu=i}^{i} \epsilon_{\nu}
$$

This $\delta_{n}$ is a slight variation of an ingenious function introduced in (1), the difference being that in (1) there is no upper restriction on $j$. We have, for every non-negative $n$,

$$
\sum_{k=0}^{n} \delta_{k} \leqslant C \sum_{k=0}^{n_{n}} \epsilon_{k}
$$

where $C$ is an absolute constant. For, for some $i_{k}, j_{k}$, with $i_{k} \leqslant k \leqslant j_{k} \leqslant k_{0}, k=$ 
$0,1,2, \ldots$,

$$
\begin{aligned}
\sum_{k=0}^{n} \delta_{k} & =\sum_{k=0}^{n}\left(j_{k}-i_{k}+1\right)^{-3 / 2} \sum_{\nu=i_{k}}^{j_{k}} \epsilon_{\nu} \\
& \leqslant \sum_{k=0}^{n} \sum_{\nu=i_{k}}^{i_{k}} \epsilon_{\nu}(|\nu-k|+1)^{-3 / 2} \\
& \leqslant \sum_{\nu=0}^{n_{0}} \epsilon_{\nu} \sum_{k=0}^{n}(|\nu-k|+1)^{-3 / 2} \\
& \leqslant C \sum_{\nu=0}^{n_{0}} \epsilon_{\nu},
\end{aligned}
$$

where $C=1+2 \sum_{k=2}^{\infty} k^{-3 / 2}$.

With these definitions we have

Lemma. For any positive $r$ let $p=p(N(r))$ be such that $N(r)=\lambda_{p}$. In each of the cases (i), (ii) and (iii) there is a sequence $\left(c_{n}\right)$, with $c_{n} \rightarrow \infty$ as $n \rightarrow \infty$, for which

$$
N\left(r e^{ \pm c_{p} \delta_{p}}\right)=N(r)
$$

for $r$ outside a set $E$, where $E$ has finite logarithmic measure in case (i) and zero lower logarithmic density in cases (ii) and (iii).

As the proof in all three cases is basically one we will prove only case (iii), which involves slightly more complications than the others.

Since $f$ is of zero order,

$$
\frac{\log N(r)}{\log r}=\eta(r) \rightarrow 0 \quad \text { as } r \rightarrow \infty .
$$

Moreover

$$
\lim _{n \rightarrow \infty}\left(\log \lambda_{n}\right)^{-1} \sum_{k=0}^{n} \delta_{n}<\infty
$$

For we know that there is a sequence $\left(m_{n}\right)$ of positive integers such that

$$
\left(\log \lambda_{m_{n}}\right)^{-1} \sum_{k=0}^{m_{n}} \epsilon_{k}=O(1) \quad \text { as } n \rightarrow \infty .
$$

Let $q_{n}$ be the largest positive integer such that $\lambda_{q_{n}-1} \leqslant \frac{1}{2} \lambda_{m_{n}}$. Then, from the definition of $n_{0}$ given by (3.2), we have $\left(q_{n}-1\right)_{0} \leqslant m_{n}$, and so from (3.4)

$$
\sum_{k=0}^{a_{n}-1} \delta_{k} \leqslant C \sum_{k=0}^{m_{n}} \epsilon_{k} .
$$

Also it follows from the definition of $\delta_{k}$ that $0 \leqslant \delta_{k}<1, k=0,1,2, \ldots$, so

$$
\sum_{k=0}^{q_{n}} \delta_{k} \leqslant 1+C \sum_{k=0}^{m_{n}} \epsilon_{k} .
$$


But $\lambda_{q_{n}}>\frac{1}{2} \lambda_{m_{n}}$, so from this together with (3.8) we obtain

$$
\begin{aligned}
\left(\log \lambda_{a_{n}}\right)^{-1} \sum_{k=0}^{q_{n}} \delta_{k} & \leqslant\left(\log \lambda_{m_{n}}-\log 2\right)^{-1}\left\{1+C \sum_{k=0}^{m_{n}} \epsilon_{k}\right\} \\
& =O(1) \text { as } n \rightarrow \infty .
\end{aligned}
$$

Thus (3.7) holds and there is an increasing sequence $\left(q_{n}\right)$ of positive integers and a constant $K$ such that

$$
\sum_{k=0}^{a_{n}} \delta_{k} \leqslant K \log \lambda_{q_{n}}, \quad n=1,2,3, \ldots
$$

Let $\lambda_{M_{n}}$ be the smallest value of the central index such that $\lambda_{q_{n}} \leqslant \lambda_{M_{n}}$ and let $R_{n}$ be such that

$$
N\left(R_{n}\right)=\lambda_{M_{n}} \text { and } N(r)<\lambda_{M_{n}} \text { for } r<R_{n}
$$

With $\eta(t)$ as given by (3.6), we can find a sequence $\left(c_{k}\right)$ with $c_{k} \rightarrow \infty$ as $k \rightarrow \infty$ for which, at least for a subsequence,

$$
\sum_{k=0}^{q_{n}} c_{k} \delta_{k} \leqslant K \eta\left(R_{n}\right)^{-1 / 2} \log \lambda_{q_{n}}, \quad n=1,2,3, \ldots,
$$

and since $\log \lambda_{q_{n}} \leqslant \log \lambda_{M_{n}}=\log N\left(R_{n}\right)=\eta\left(R_{n}\right) \log R_{n}$, we obtain from (3.9)

$$
\sum_{k=0}^{q_{n}} c_{k} \delta_{k} \leqslant K\left\{\eta\left(R_{n}\right)\right\}^{1 / 2} \log R_{n}
$$

We seek to determine the size of the subset of $\left[1, R_{n}\right]$ at the points of which

$$
N\left(r e^{c_{p} \delta_{p}}\right)=N(r)
$$

where $p$ is such that $N(r)=\lambda_{p}, p=p(N)$.

Let $r_{1}$ be the first number no less than 1 for which $(3.11)$ is false and let $r_{1}^{\prime}=r_{1} e^{c_{p} p_{1} p_{1}}$, where $p_{1}=p\left(N\left(r_{1}\right)\right)$. Let $r_{2}$ be the smallest number no less than $r_{1}^{\prime}$ at which (3.11) is false and let $r_{2}^{\prime}=r_{2} e^{c p_{2} \delta} p_{2}$, where $p_{2}=p\left(N\left(r_{2}\right)\right)$. We continue in this way until reaching $r_{s}$ where $s$ is such that $r_{s}<R_{n}$ and $r_{s+1} \geqslant R_{n}$.

We note that $N\left(r_{i}\right) \geqslant N\left(r_{i-1}^{\prime}\right)>N\left(r_{i-1}\right)$, so the sequence $\left(p_{i}\right)_{i=1}^{s}$ is strictly increasing. Also, since $r_{s}<R_{n}, N\left(r_{s}\right)<\lambda_{M_{n}}$ and so, from the definition of $M_{n}, p\left(N\left(r_{s}\right)\right)=p_{s}<q_{n}$. Thus (3.11) holds in $\left[1, R_{n}\right]$ outside a subset of logarithmic measure at most

$$
\sum_{i=1}^{s} c_{p_{i}} \delta_{p_{i}} \leqslant \sum_{k=0}^{q_{n}} c_{k} \delta_{k} \leqslant K\left\{\eta\left(R_{n}\right)\right\}^{1 / 2} \log R_{n}
$$

In a precisely similar way, but defining the sequences $\left(r_{i}\right),\left(r_{i}^{\prime}\right)$ to be decreasing from $R_{n}$, we see that

$$
N\left(r e^{-c_{p} \delta_{p}}\right)=N(r)
$$

where $p$ is such that $N(r)=\lambda_{p}$, also holds in $\left[1, R_{n}\right]$ outside a subset of logarithmic measure at most $K\left\{\eta\left(R_{n}\right)\right\}^{1 / 2} \log R_{n}$. On combining this with (3.11) and (3.12) we see that (3.11) and (3.13) hold together in [1, $\left.R_{n}\right]$ outside a subset of logarithmic measure at $2 K\left\{\eta\left(R_{n}\right)\right\}^{1 / 2} \log R_{n}$. Since $R_{n} \rightarrow \infty$ as $n \rightarrow \infty$ and $\eta(t) \rightarrow 0$ as $t \rightarrow \infty$, the case (iii) of the Lemma is proved. 


\section{Proof of the result}

With $p$ such that $N(r)=\lambda_{p}$, let us consider

$$
S(r)=\sum_{n=0}^{p-1}\left|a_{n}\right| r^{\lambda_{n}}+\sum_{n=p+1}^{\infty}\left|a_{n}\right| r^{\lambda_{n}}
$$

It is known (see for example Lemma 2 of (2)) that, for $r$ outside a set $F$ of finite logarithmic measure and for $n_{0}$ as given by (3.2),

$$
\sum_{n=p_{o}+1}^{\infty}\left|a_{n}\right| r^{\lambda_{n}}=o(\mu(r)) \quad \text { as } r \rightarrow \infty .
$$

Hence, as $r$ tends to infinity outside $F$,

$$
S(r)=\sum_{n=0}^{p-1}\left|a_{n}\right| r^{\lambda_{n}}+\sum_{n=p+1}^{p_{0}}\left|a_{n}\right| r^{\lambda_{n}}+o(\mu(r)) .
$$

Let $r$ be a number outside $F \cup E$, where $E$ is the exceptional set of the Lemma. For $p+1 \leqslant n \leqslant p_{0}$, we obtain from one half of the Lemma,

so that

$$
\left|a_{n}\right|\left\{r e^{c_{p} \delta_{p}}\right\}^{\lambda_{n}} \leqslant\left|a_{p}\right|\left\{r e^{c_{p} \delta_{p}}\right\}^{\lambda_{p}}
$$

$$
\left|a_{n}\right| r^{\lambda_{n}} \leqslant \mu(r) e^{-\left(\lambda_{n}-\lambda_{p}\right) c_{p} \delta_{p}} .
$$

Also, for $p+1 \leqslant n \leqslant p_{0}$ we obtain, with two applications of the arithmetic-geometric means inequality (following (1)),

$$
\begin{aligned}
\delta_{p} & \geqslant(n-p)^{-3 / 2} \sum_{\nu=p}^{n-1} \frac{1}{\lambda_{\nu+1}-\lambda_{\nu}} \\
& \geqslant(n-p)^{-1 / 2}\left\{\prod_{\nu=p}^{n-1} \frac{1}{\lambda_{\nu+1}-\lambda_{\nu}}\right\}^{1 /(n-p)} \\
& \geqslant(n-p)^{1 / 2}\left(\lambda_{n}-\lambda_{p}\right)^{-1} .
\end{aligned}
$$

Hence, for $p+1 \leqslant n \leqslant p_{0}$,

$$
\left|a_{n}\right| r^{\lambda_{n}} \leqslant \mu(r) e^{-c_{p}(n-p)^{1 / 2}}
$$

For $0 \leqslant n \leqslant p-1$ we employ the remaining half of the Lemma to obtain, quite analogously,

$$
\left|a_{n}\right| r^{\lambda_{n}} \leqslant \mu(r) e^{-c_{p}(p-n)^{1 / 2}}
$$

Thus, as $r$ tends to infinity outside $F \cup E$,

$$
\begin{aligned}
S(r) & \leqslant 2 \mu(r) \sum_{n=1}^{\infty} e^{-c_{p} n^{1 / 2}}+o(\mu(r)) \\
& =o(\mu(r))
\end{aligned}
$$

The result follows.

\section{REFERENCES}

(1) P. ERDös and A. J. Macintyre, Integral functions with gap power series. Proc. Edinburgh Math. Soc. (2) 10 (1953), 62-70. 
(2) W. K. HAYMAN, The local growth of power series: a survey of the Wiman-Valiron method by, Canad. Math. Bull. 17 (1974), 317-358.

\section{UNIVERSITY OF OTAGO}

BOX 56, DUNEDIN

NEW ZEALAND 